\title{
Compulsory Insurance and Consumer Welfare: a State-Claims Approach*
}

\author{
by Harris Schlesinger**
}

\section{Introduction}

The notion that insurance markets may not be complete is beginning to gain some acceptance in academic circles. ' Real-world frictions can sometimes be the culprit that interferes with the free exchange of insurance contracts. One small piece of the ever-growing mosaic is presented by Schulenburg [1986], who considers possible effects of government intervention into consumer insurance-purchasing decisions. In particular, Schulenburg considers how compulsory coverage in one insurance line might affect insurance decisions in other lines, and the resulting effects on consumer welfare. The purpose of this paper is to illustrate Schulenburg's welfare results with a simple state-claims diagram and to offer some new welfare results, which follow easily from Schulenburg's model.

\section{The model}

We simplify Schulenburg's model to a two-state framework so that his results can be modelled via a state-claims diagram. The model considers a risk averse consumer with random wealth consisting of two components,

$$
\tilde{Y}=\tilde{L}+\tilde{W} .
$$

* This paper is an extension of the author's comments on Matthias Schulenburg's paper at the llth Seminar of the European Group of Risk and Insurance Economists, Geneva, September 19-21, 1984. Thanks are due to participants of the Seminar for helpful comments.

** International Institute of Management, Berlin.

${ }^{\prime}$ A fair number of recent articles have addressed this issue and many additional papers on related topics are currently in process. For an overview of this literature, see Schlesinger and Doherty [1985]. 
In the above notation, “ " denotes a random variable. For our purposes, we consider

$$
\tilde{L}=\left\{\begin{array}{l}
-L \text { with the probability } p_{L} \\
-0 \text { with probability } 1-p_{L}
\end{array}\right.
$$

and

$$
\tilde{\mathrm{W}}=\left\{\begin{array}{cc}
M-W \text { with probability } p_{W} \\
M & \text { with probability } 1-p_{W}
\end{array}\right.
$$

Thus, the individual can be thought of as possessing an initial wealth of $M$ and facing possible losses of amounts $L$ and $W$. We assume that insurance is available at any desired level of coverage on both losses and that insurance premiums equal actuarial value plus a proportional loading.

We next consider government's intervention by specifying a compulsory level of coverage for insurance against loss $W$. In the results that follow, it is assumed that the compulsory level of insurance specified by the government must be net by purchasing that exact amount of insurance coverage. If the compulsory level of coverage is only considered to be a minimum allowable level of coverage, so that the consumer is free to purchase more than the compulsory amount, some of the propositions below would need to be modified. But, this modification is straightforward and is left to the reader.

\section{Welfare results}

Although compulsory coverage is likely to affect consumer welfare under many circumstances, we only consider two very extreme cases for the purpose of illustration. Namely, we consider the cases where $\tilde{W}$ and $\tilde{L}$ exhibit a perfect positive or negative correlation and the sizes of the two losses are equal; i.e., $L=W^{2}$ The following Propositions are proved by Schulenburg:

Proposition $A$ : If all insurance premiums are actuarially fair and $\tilde{W}$ and $\tilde{L}$ exhibit perfect negative correlation, then any increase in compulsory insurance coverage will not affect the consumer's welfare.

Proposition $B$ : If the premium loadings are equal for both insurance markets, and $\tilde{W}$ and $\tilde{L}$ exhibit perfect positive correlation, then an increase in compulsory coverage will not affect the consumer's welfare so long as the consumer's original insurance coverage against $L$ is at least as great as the increase in the compulsory level.

Under the hypothesis of proposition A, note that there is no risk in the individual's initial wealth position. The individual always experiences a loss of $W$ or a loss of $L$, but not both simultaneously; and since we assume $L=W$, initial wealth is nonstochastic. It is easy to show that no insurance coverage is optimal if no compulsory insurance is required. (See Doherty and Schlesinger [1983]) This is seen easily in figure 1.

${ }^{2}$ Schulenburg's hypotheses are slightly more general, assuming perfect correlation and equal variances of $\tilde{L}$ and $\tilde{W}$. Also, our Propositions $\mathrm{A}$ and $\mathrm{B}$ below correspond to Propositions 6 and 7 of Schulenburg respectively. 


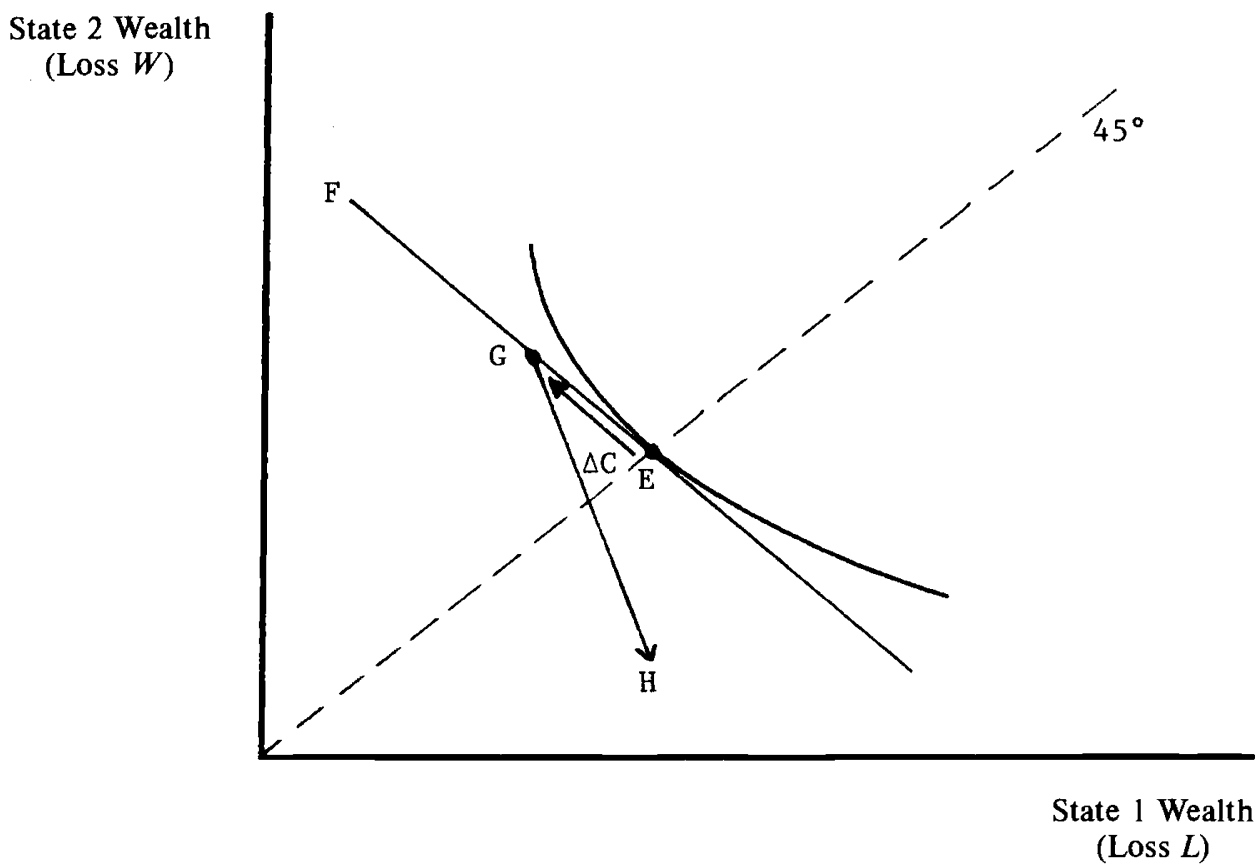

Figure 1

In figure 1, we depict the consumer's contingent wealth claims in the two possible states of the world:

State 1 - in which loss $L$ occurs

and

State $2-$ in which loss $W$ occurs.

The consumer's initial contingent wealth claim, $E$, lies along the $45^{\circ}$ certainty line since $L=W$. Line $E F$ depicts the so-called fair-price line. As we move from $E$ towards $F$ along this line, we are purchasing insurance against loss $W$ at an actuarially-fair price. If the government were to change the amount of compulsory coverage from zero to an amount $\Delta C$, the consumer would be worse off (at point $G$ ) absent any compensating change in coverage against loss $L$. Since coverage is available at an actuarially-fair price, the consumer would purchase an equal amount of insurance against loss $L$, moving from $G$ back to point $E$. Consequently, there is no change in the consumer's welfare. 
Note that this would not be the case if a positive loading was added to the premium for coverage on $\tilde{L}$. This is true regardless of whether or not the premium for coverage on $\tilde{W}$ contains a loading. For the sake of concreteness, let coverage on $\tilde{W}$ be priced at its actuarial value and suppose the government increases the level of compulsory coverage by amount $\Delta C$. Thus, the consumer is initially moved from point $E$ to point $G$ in figure 1. But now the consumer is allowed to buy insurance on $\tilde{L}$ at a price including a positive proportional loading, such as along ray $G H$. Obviously, in this case, consumer welfare will be reduced as a consequence of the increased level of compulsory coverage. ${ }^{3}$

We can illustrate Proposition B in a similar manner. Under the hypothesis of Proposition B, either both losses occur, or neither loss occurs. Thus the two potential states of the world are:

State 1 - no losses occur

and

State 2 - both losses occur.

This is illustrated in figure 2 .

State 2 Wealth

(Loss)

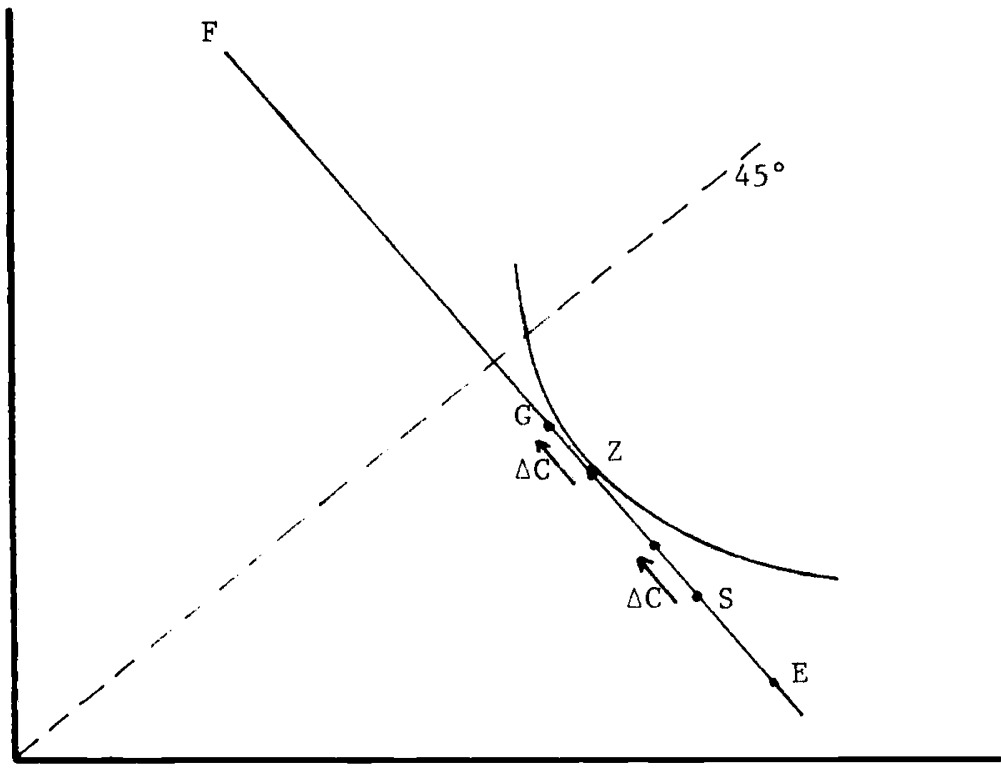

State 1 Wealth

(No loss)

Figure 2

\footnotetext{
${ }^{3}$ This analysis assumes that only nonnegative insurance indemnities are possible. The same result of reduced welfare also holds if we allow the posibility of negative insurance indemnities - which would be analagous to writing a put option - but this goes beyond the scope of the present paper. Interested readers can develop this result themselves by extending section $V c$ of Schlesinger and Doherty [1985].
} 
In figure 2, point $E$ again denotes the endowed contingent-wealth claim. We now assume that the consumer is achieving the claim denoted $Z$ by purchasing insurance on $\tilde{W}$ to $S$ and insurance on $\tilde{L}$ from $S$ to $Z$. Insurance prices are depicted by the line $E F$, which as drawn shows equal positive loadings. Proposition $B$ is illustrated by supposing that insurance on $\tilde{W}$ is compulsory and increased by an amount $\Delta C$. With no change in coverage on $\widetilde{L}$, the consumer would experience a welfare loss by holding contingent claim $G$. However, a reduction in coverage on $\tilde{L}$ by an amount equal to $\Delta C$ will move the consumer from $G$ back to point $Z$. Consequently, there is no change in consumer welfare as stated in Proposition B.

Suppose now that the loadings on the two coverages are unequal. In this case we can show that a definite welfare loss or gain will arise, depending upon which loading is greater. If the loading for coverage on $\tilde{W}$ is greater, there will be a welfare loss from increasing the level of compulsory coverage. This is shown in figure 3.

State 2 Wealth

(Loss)

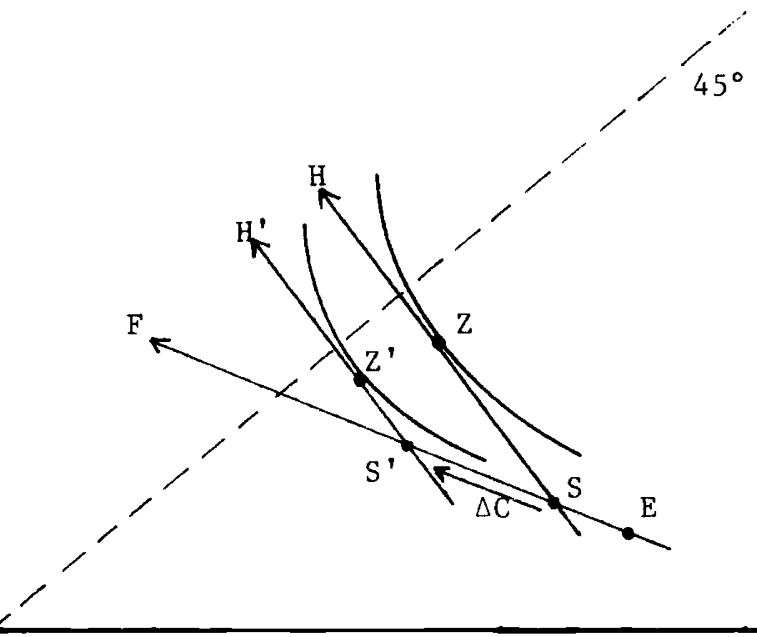

State 1 Wealth

(No Loss)

Figure 3

In figure 3, $E$ and $S$ are as in figure 2. But, now we assume that insurance on $\tilde{L}$ available at a premium with a lower loading factor. The price of insurance on $\tilde{L}$ is characterized by the slope of line $S H$, which is steeper than line $E F$, indicating a lower reduction in state 1 wealth per net indemnity dollar in state 2 . Initially, we depict the consumer as purchasing insurance on $\tilde{L}$ 
sufficient to maintain contingent claim $Z$. However, we see that if compulsory coverage is increased by an amount $\Delta C$ to $S^{\prime}$, the individual must take a reduction in welfare, $Z^{\prime}$ being the best achievable contingent claim.

Although we do not show it here, a similar analysis easily demonstrates that consumer welfare will increase when the loading for insurance on $\tilde{L}$ is higher than the loading for insurance on $\tilde{W}$.

\section{Concluding remarks}

We have demonstrated Proposition A and B, due to Schulenburg, in a simple two-state model. It was fairly easy to extend these results to show conditions under which consumer welfare would change. The intuition behind these results is quite simple. In the case of perfect negative correlation, the initial contingent wealth position is riskless. Any compulsory coverage on $\tilde{W}$ enacted by the government introduces risk where none existed before. With actuarially fair prices, this risk can be totally eliminated by purchasing an equal amount of insurance on $\tilde{L}$. However, if insurance on $\tilde{L}$ includes a positive loading, we cannot return to the initial position and must suffer a welfare loss.

For the case where $\tilde{W}$ and $\tilde{L}$ are perfectly correlated, we essentially have a potential loss of size $W+L$ that either does or does not occur. If insurance on $\tilde{W}$ and $\tilde{L}$ sell for the same premium, any compulsory increase in coverage on $\tilde{W}$ is effectly counteracted by an equivalent reduction of coverage on $\tilde{L}$. If the prices differ, the consumer would like to buy as much insurance as is possible at the cheaper of the two prices. Since we take insurance on $\tilde{W}$ to be compulsory, any increase in compulsory coverage will increase (decrease) consumer welfare if coverage on $\tilde{W}$ is the cheaper (more expensive) of the two coverages.

\section{REFERENCES}

DOHERTY, N. and SCHLESINGER, H. [1983]: "The optimal deductible for an insurance policy when initial wealth is random", Journal of Business, 56 (October 1983), 555-565.

SCHLESINGER, H. and DOHERTY, N. [1985]: "Incomplete markets for insurance: an overview", Journal of Risk and Insurance, 52 (September 1985), 402-423.

SCHULENBURG, J.-M.Gf.v.d. [1986]: "Optimal insurance purchasing in the presence of compulsory insurance and uninsurable risks", Geneva Papers on Risk and Insurance, this issue. 